\title{
LIPOSOMES AS AN OCULAR DELIVERY SYSTEM FOR FLUCONAZOLE: IN-VIVO STUDY
}

F. S. Habib ${ }^{1}$, E. A. Fouad ${ }^{1}$, M. S. Abdel-Rahman ${ }^{2}$ and Dina Fathalla ${ }^{1}$

${ }^{1}$ Department of Pharmaceutics, Faculty of Pharmacy, Assiut University, Assiut, Egypt

${ }^{2}$ Department of Ophthalmology, Faculty of Medicine, Assiut University, Assiut, Egypt

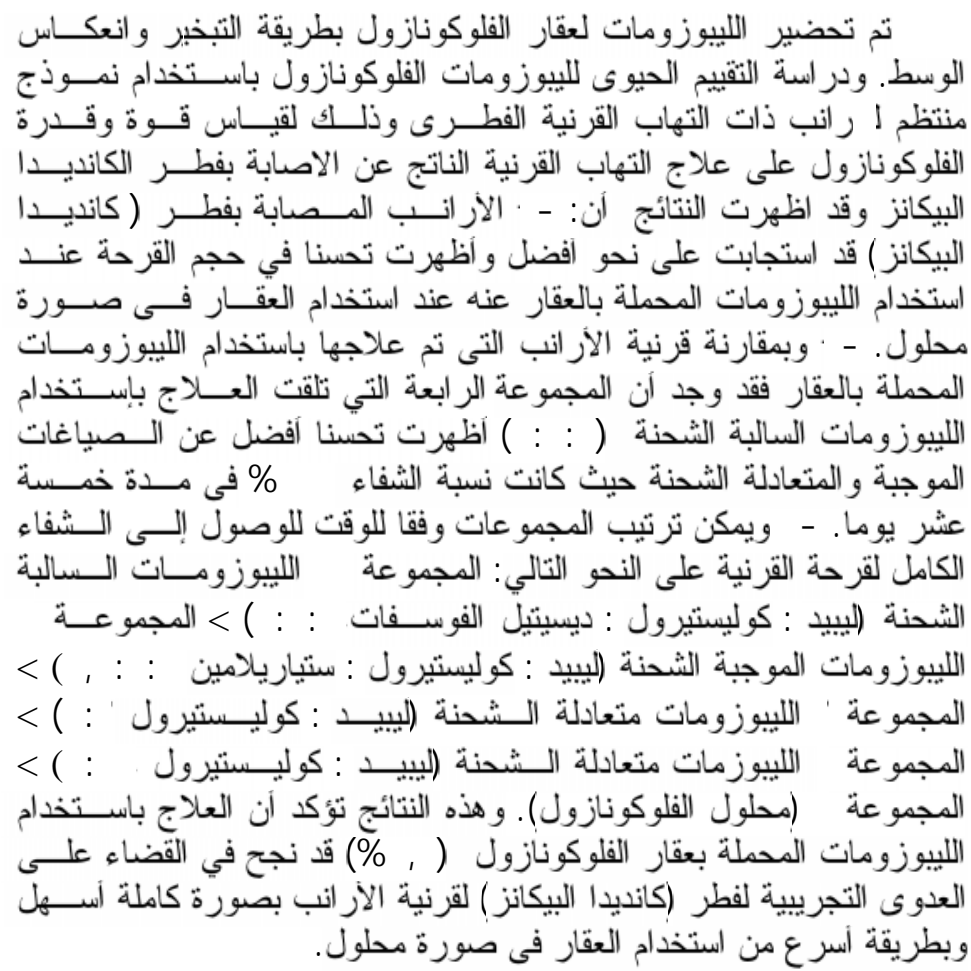

The purpose of this study was to formulate topically effective controlled release ophthalmic fluconazole liposomal formulations using the reverse-phase evaporation technique. Soya bean phosphatidylcholine $(P C)$ and cholesterol $(C h)$ in specific weight ratios were used. Selected formulations were tested for their in-vivo ocular antifungal effect. These included the neutral, the positively (using stearyl amine) and the negatively (using dicetyl phosphate) 
charged liposomes. A reproducible model of Candida keratitis in rabbits was performed and the effects of the prepared liposomes were better than a solution of fluconazole. The order of fluconazole liposomal formulations according to the time to achieve complete healing is arranged in a descending order: negatively charged liposomes > positively charged liposomes > neutral liposomes (7:4) > neutral liposomes (5:5) > fluconazole solution. The frequency of instillation was decreased; also, the time of ulcer healing was decreased. It was concluded that the use of liposomes as a drug delivery system could contribute to the enhancement of the effect of fluconazole in the eye.

\section{INTRODUCTION}

Drug delivery in ocular therapeutics is a challenging problem and is a subject of interest to scientists working in the multidisciplinary areas pertaining to the eye. Current trends in ocular therapeutics and drug delivery suggest that the existing dosage forms will be replaced by novel drug delivery systems that offer improved biopharmaceutical properties ${ }^{1}$.

The treatment of fungal keratitis remains a serious and unresolved problem. Most antifungal drugs are effective against superficial mycosis but are less successful in counteracting deep mycotic infiltrations. In particular, the treatment of keratomycosis is often frustrating owing to limited tissue penetration, narrow antimicrobial spectrum, and toxicity of the antifungal agents currently available. In recent decades, many experimental and clinical studies have shown fluconazole to be safe and effective antifungal agent for the topical treatment against deep keratitis $^{2-5}$.
Fluconazole is the first of a new subclass of synthetic triazole antifungal agents with broad spectrum activity. It is primarily fungistatic with activity against Blastomyces dermatitidis, Candida spp ${ }^{6}$. Coccidioides immitis, Cryptococcus neoformans, Epidermophyton, spp., Histoplasma capsulatum, Microsporues spp., and Trichophyton spp. Fluconazole was approved for systemic candidiasis, oropharyngeal and esophageal candidiasis ${ }^{7-10}$. In sensitive fungi fluconazole is acting through the inhibition of Cytochrome $\mathrm{P}-450$ dependent enzymes resulting in impairment of ergosterol synthesis in fungal cell membrane ${ }^{11}$. Fluconazole offers many advantages such as its water solubility, low level of protein binding, high bioavailability, long half-life, metabolic stability, and high body tissues and fluid penetrations $^{11 \& 12}$. The high penetration into the aqueous humour and low toxicity of fluconazole makes it a good candidate for consideration as a topical ocular antifungal $\operatorname{agent}^{13 \& 14}$. 
The penetration of drug molecules into the eye from a topically applied preparation is a complex phenomenon. The rate of drug penetration depends not only on the physicochemical properties of the drug itself, such as its solubility and particle size, in case of the suspensions, but also on those of its vehicle ${ }^{15}$.

In the liposomal dosage form the drug is encapsulated in to lipid vesicles, which can cross cell membranes. The liposomes, therefore, can be viewed as drug carriers, and as such, they can change the rate and extent of absorption, as well as the disposition of the drug. As yet there is not much known about the mechanism by which liposomes interact with the cornea ${ }^{16}$.

Various possible mechanisms by which liposomes can interact with the cells include lipid exchange, stable adsorption, endocytosis and fusion ${ }^{16}$. It is not possible to assign a single mechanism in a given actual in-vivo system, as a combination or any one of these may be responsible in that experimental situation. Endocytosis usually occurs in the cells capable of phagocytosis. Consequently, this mechanism cannot be the dominant one here. Fusion of the liposomes with the cells requires special conditions of lipid fluidity, temperature and is more prominent in the presence of certain chemical agents. Fusion of liposomes with the cornea may exist to some extent, but the major mechanism might be the adsorption and / or surface lipid exchange $^{16}$. If the liposomes were taken up as such, absorbed as intact vesicles containing their drug, then the ocular drug concentration would be similar irrespective of the nature of the drug entrapped; the absorption would only depends on the type of liposomes, their size and surface charge $^{16}$. It is difficult to ascertain whether intact liposomes penetrate through the cornea, or fuse with the corneal cells, or remain in the conjunctival sac and interact with tear components leading to gradual release of the entrapped drug which is then absorbed by the cornea. The ability of drugs to diffuse into the corneal epithelium is influenced by its partition coefficient. The epithelium has been demonstrated to be a greater barrier to hydrophilic rather than lipophilic compounds ${ }^{17}$.

The objective of this work was to prepare a liposomal fluconazole corneal drug delivery system for the purpose of increasing contact time and prolonging antifungal action of the drug in comparison to fluconazole solution. A reproducible model of Candida keratitis in rabbits was performed to study the antifungal activity of selected fluconazole liposomal formulations compared with that of fluconazole solution.

\section{EXPERIMENTAL}

\section{Materials}

- Fluconazole, (Kindly provided by CID Co., (Cairo, Egypt).

- Phosphatidylcholine from Soyabean (PC), Cholesterol (Ch), Stearylamine (SA), and Dicetyl 
phosphate (DP) were purchased from Sigma Chemical Co., (St. Louis, USA,).

- Methyl alcohol was obtained from BDH Ltd., (Poole, U.K.).

- Chloroform, diethyl ether, sodium hydrogen phosphate, disodium hydrogen phosphate and sodium chloride were purchased from Adwic, El-Nasr Pharmaceutical Co., (Cairo, Egypt). All reagents were of analytical grade and $99 \%$ pure.

- Double distilled water, boiled and cooled was used throughout the experiments.

- Disposable syringe filter $0.22 \&$ 0.45 m uni $\mathrm{FlO}^{\circledR-} 25$ Schleicher \& Schuell Inc., (Keene NH 03431 USA.).

- Thiopental $^{\circledR} \quad(0.5 \quad \mathrm{gm} / 10 \quad \mathrm{ml})$ Biochemie GmbH, (ViennaAustria).

- Inoculum: Candida albicans No. 4925 were used for animal inoculation. (Supplied from Mycological center, Faculty of Science Assiut University) previously isolated from cases of keratomycosis.

- Experimental animals: Forty male healthy, rabbits weighing approximately $(1.5-2.5 \mathrm{Kg})$ each were used.

\section{Equipment}

- Rotavapor, type R 110, [Buchi, Switzerland].

- Waterbath, Buchi 462, [Buchi, Switzerland].
- Sensitive electric balance, [Precisa 205A Super Bal-Series, Swiss Quality].

- Sonicator, Model 275T, Crest Ultrasonics Corp., [Trenton, USA].

- Refrigerated centrifuge, Model 8880, Centurion Scientific Ltd., [W. Sussex, U.K.].

- Laminar air flow hood, Model NB 48 INOX Gelaire Class 100, Gelman Instruments.

\section{Methodology}

Preparation of liposomes

All the steps were performed under aseptic conditions. All glassware were sterilized by heating in hot air oven over $120^{\circ} \mathrm{C}$ for 2 hours. Boiled double distilled water was passed through a 0.22- $\mathrm{m}$ disposable syringe filter (bacterial filter), and the entire procedures were performed under laminar air flow hood in presence of flame. Fluconazole liposomes were prepared using the reverse-phase evaporation technique $^{18}$.

The lipid components (phosphatidylcholine and cholesterol either alone or mixed with charge inducing agent such as stearylamine or dicetyl phosphate) expressed as weight ratios $^{19 \& 20}$ of the selected formulas are presented in Table 1. The different liposomal ingredients equivalent to $50 \mathrm{mg}$, were weighed into $250 \mathrm{ml}$ long-necked quick fit round bottom flask and dissolved in $10 \mathrm{ml}$ chloroform. The organic solvent was slowly evaporated under reduced pressure, using a rotary evaporator, at $40^{\circ} \mathrm{C}$ to roduce a thin 
Table 1: Fluconazole liposomal formulations expressed as weight ratios of lipid components.

\begin{tabular}{|c|c|c|c|c|}
\hline $\begin{array}{c}\text { Liposome } \\
\text { formulae }\end{array}$ & $\begin{array}{c}\text { Phosphatidyl } \\
\text { choline (PC) }\end{array}$ & Cholesterol $(\mathrm{Ch})$ & Stearylamine (SA) & $\begin{array}{c}\text { Dicetyl } \\
\text { phosphate } \\
\text { (DP) }\end{array}$ \\
\hline 1 & $7(31.82 \mathrm{mg})$ & $4(18.18 \mathrm{mg})$ & - & - \\
\hline 2 & $5(25 \mathrm{mg})$ & $5(25 \mathrm{mg})$ & - & - \\
\hline 3 & $5(22.72 \mathrm{mg})$ & $5(22.72 \mathrm{mg})$ & & $1(4.54 \mathrm{mg})$ \\
\hline 4 & $5(23.8 \mathrm{mg})$ & $5(23.8 \mathrm{mg})$ & $0.50(2.38 \mathrm{mg})$ & \\
\hline
\end{tabular}

lipid film. The lipid film was redissolved in $10 \mathrm{ml}$ ether. Fluconazole solution in $10 \mathrm{ml}$ acetone together with $5 \mathrm{ml}$ distilled water was then added. The mixture was sonicated for one minute, swirled by hand, and resonicated for another minute. The organic solvents were evaporated on the rotary evaporator under reduced pressure. The liposomal suspension was kept overnight in the refrigerator at $5^{\circ} \mathrm{C}$ to mature.

\section{Preparation of fluconazole loaded liposomes eye drops}

Fluconazole eye drops were prepared under aseptic condition by diluting the optimized liposomes preparations with Sørensen`s modified phosphate buffer $\mathrm{pH}=7$ containing $0.01 \%$ benzalkonium chloride as preservative so that, the eye drops contained the equivalent amount of $0.2 \%$ of the drug.

Four liposome formulations were considered:

* Neutral liposomes PC:Ch weight ratio 7:4.

* Neutral liposomes PC:Ch weight ratio 5:5.

$\begin{array}{lcl}\text { * Negatively } & \text { charged } & \text { liposomes } \\ \text { PC:Ch:DP } & 5: 5: 1 . & \\ \text { * Positively } & \text { charged } & \text { liposomes } \\ \text { PC:Ch:SA } & 5: 5: 0.5 & \end{array}$

In-vivo antifungal evaluation (Experimental Candida keratitis in rabbits)

Animals: Forty adult rabbits were used in this study. Animals received standard dry food pellets and water. All eyes were initially examined by an ophthalmologist with hand-held torch. Only animals without any signs of ocular pathology were included.

Yeast: Candida albicans strain No. 4925 was used for all experiments. This well-characterized strain has been used in a rabbit keratitis model, in which it proved (experimentally in Mycological center) to be highly invasive for the corneal stroma after surface inoculation.

\section{Inoculation technique}

The procedure is based on a model of experimental keratomycosis ${ }^{21}$. The rabbits were sedated by the intraperitoneal injection of $0.5 \mathrm{ml}$ thiopental. ${ }^{\circledR}$ Intrastromal injection of $10 \quad 1$ of 
inoculum (Candida albicans, containing $2.5 \times 10^{5}$ cell), was done in both eyes by inserting a sterile 27 gauge needle into the central corneal stroma tangential to the corneal surface to a depth of one half of the corneal thickness.

The animals were excluded from the study if there was penetration of the inoculum into the anterior chamber or reflux of the inoculum was observed.

\section{Treatment procedure}

Rabbits were randomly divided into five equal groups, (eight rabbits) and the right eye of each received:

- The first group, fluconazole solution $(0.2 \% \mathrm{w} / \mathrm{w})$.

- Second group, neutral fluconazole liposomes (PC:Ch; 5:5).

- Third group, neutral fluconazole liposomes (PC:Ch; 7:4).

- Fourth group, negatively charged fluconazole liposomes (PC:Ch:DP; $5: 5: 1)$.

- Fifth group, positively charged fluconazole liposome (PC:Ch:SA; 5:5:0.5).

The left eye of each rabbit did not receive any treatment and was considered as control.

\section{Application of the eye drops and the follow up}

Eye drops were instilled $50 \mathrm{l} /$ application into the conjunctival sac of rabbits after 48 hours of the inoculation procedure. The instillation continues every 3 hours for 12 hours (four times daily) in the first three days, then every four hours (three times daily) in the next period of treatment (18 days). The rabbits eyes were examined daily over a 21 day period by hand- held torch for signs of infection, and the severity of inflammatory reaction was noted (hypopyons, iritis) by the ophthalmologist. Photographs were taken after the induction of keratitis, before treatment, during treatment period and at the end of treatment in order to find out signs of improvement. Occurrence of complications such enodophthalmitis was noted.

\section{Statistical analysis}

Statistical analysis was carried out employing one-way ANOVA tests followed by two-tailed paired Student's $t$ test. Difference at $P<0.05$ was considered as minimal level of significance.

\section{RESULTS AND DISCUSSION}

\section{In-vivo antifungal evaluation (Experimental Candida keratitis in rabbits)}

In the present study, a wellestablished rabbit model of fungal keratitis $^{22}$ was used to investigate the potential of fluconazole in the therapy of deep keratitis due to $C$. albicans. The model is reproducible without the need of immunosuppressive pretreatment. Therapy is started only on day 2, when stromal keratitis is manifested; hence the model was more closely parallel to human corneal candidiasis ${ }^{23}$.

Many experimental and clinical studies used fluconazole in solution 
as eye drops to treat deep keratitis. The eye drops were administered every 30 minutes or one hour for 8 to 10 hours for 7 to 21 days depending on the severity ${ }^{21 \& 24-26}$. To overcome this tedious process and to accomplish patient compliance, fluconazole liposomes in the present study was prepared for exerting a sustained release effect of the drug. Therefore, the liposomal formulations were instilled four times daily in the first three days then three times daily for the next period of treatment. All eyes inoculated with $C$. albicans developed corneal ulcer (oval in shape, whitish in color) on the second day (Fig. 1).

Signs of corneal healing were observed in all groups treated with either fluconazole solution or liposomal formulations with variation in percent of healing and time for reaching healing. It was found that, rabbits infected with $C$. albicans responded better and showed improvement in size of ulcer and hypopyon improved on using fluconazole liposomal formulae than using fluconazole solution.

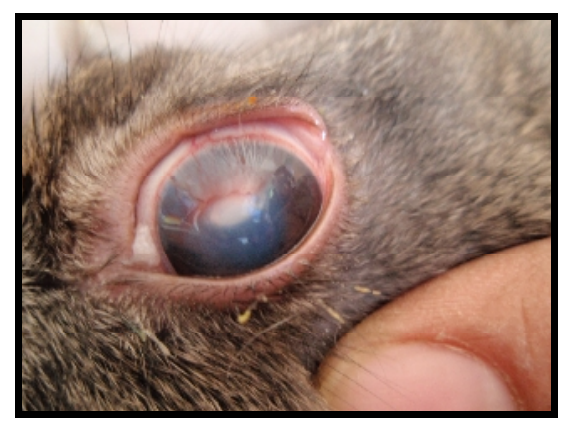

Fig. 1: Candida keratitis 48 hours following inoculation with Candida albicans.
Also, when comparing rabbits' cornea, treated with different liposomal formulations, group 4 which received fluconazole loaded negative liposome PC:Ch:DP 5:5:1 showed better improvement than positive and neutral formulations as shown in Figures (2-6).

At the end of treatment periods with fluconazole loaded liposomal preparations ulcers are resolved, leaving a non-adherent leucoma, also, hypopyon resolved and the cornea cleared from the periphery with a residual limited corneal non-adherent leucoma, Figures (2-6).

- In group1 ( fluconazole solution), the mean time of complete healing from Candida keratitis in 50\% of the rabbits was 20 days (range1921) while in the other $50 \%$ only slight improvement was noticed (Fig. 2).

- Group 2 showed complete healing after 20 days (range 18-21) representing $87.5 \%$ of the rabbits' cornea (Fig. 3).

- Group 3 showed complete healing after 17 days in (range 15-18) representing $87.5 \%$ of the rabbits' cornea (Fig. 4).

- Group 4 showed complete healing after 15 days in (range 14-16) representing $100 \%$ of the rabbits' cornea (Fig. 5).

- Group 5 showed mean times of complete healing 16 days (range 15-17) representing $87.5 \%$ of the rabbits' cornea (Fig. 6).

The groups are arranged according to the time to reach complete healing as: group $4<$ group $5<$ group $3<$ group $2<$ group 1 . 


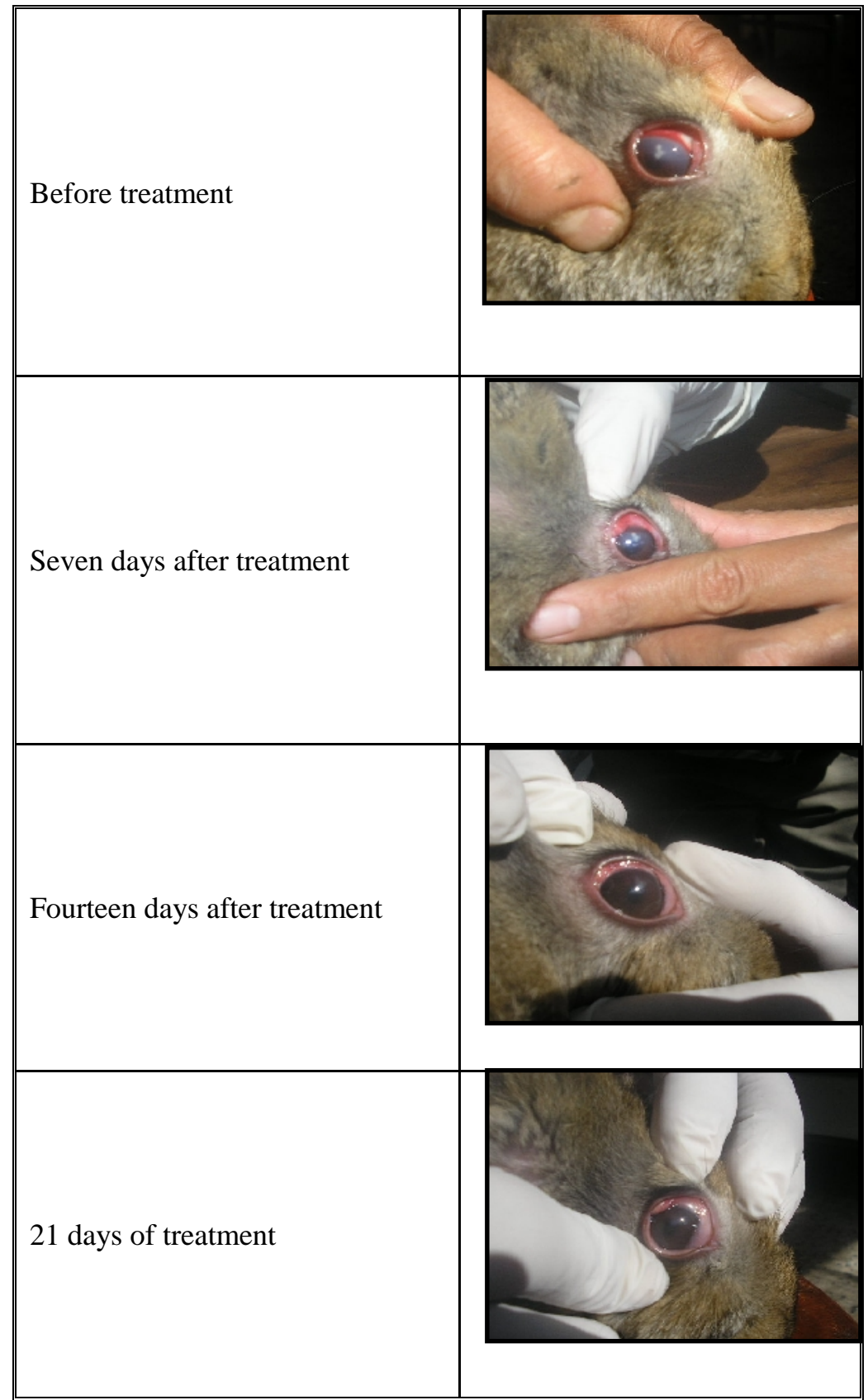

Fig. 2: Photographs showing gradual stages of healing and disappearance of Candida keratitis treated with $0.2 \% \mathrm{w} / \mathrm{w}$ fluconazole solution (first group). 


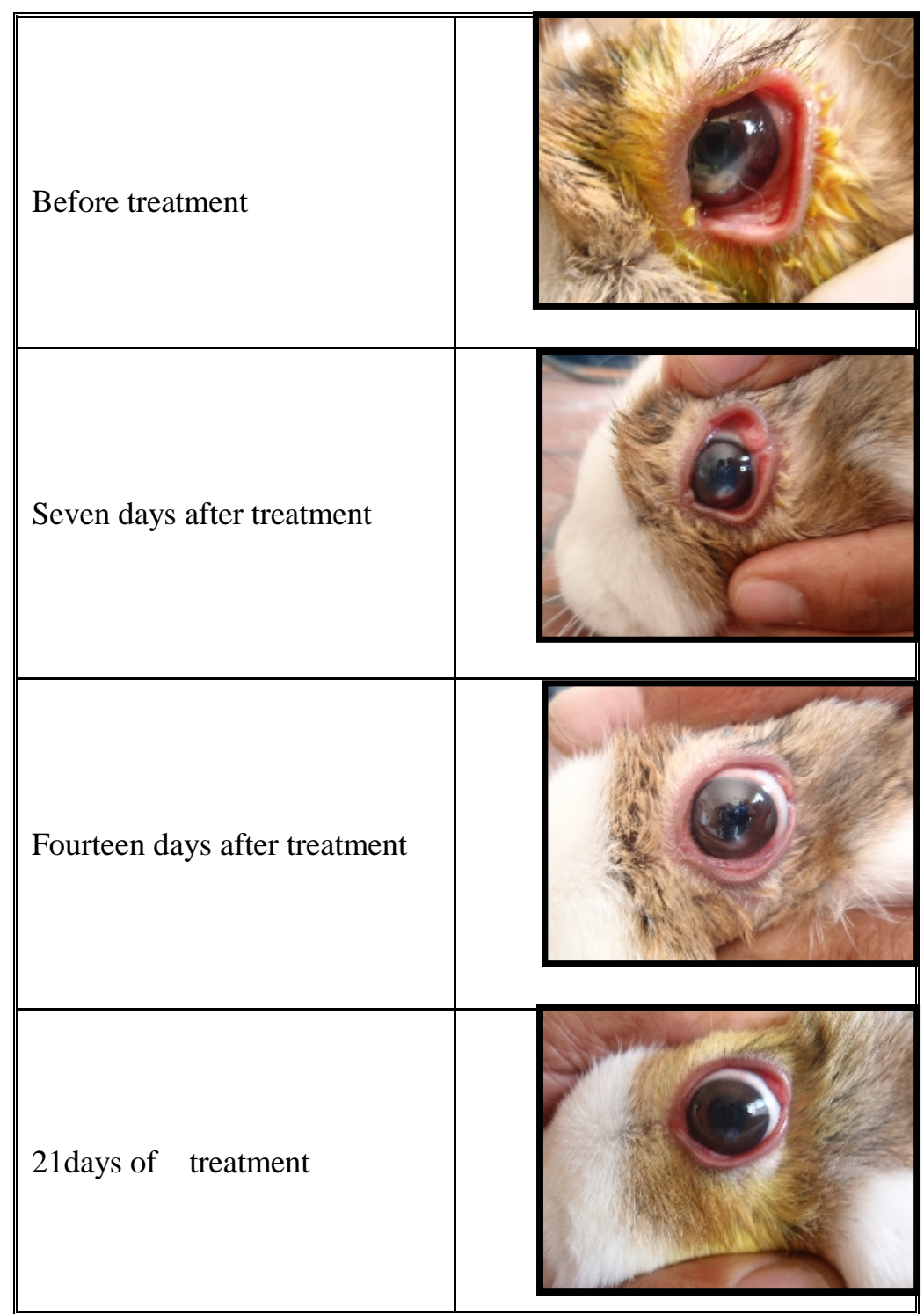

Fig. 3: Photographs showing gradual stages of healing and disappearance of Candida keratitis treated with fluconazole loaded neutral liposome composed of PC:Ch (5:5) (second group). 


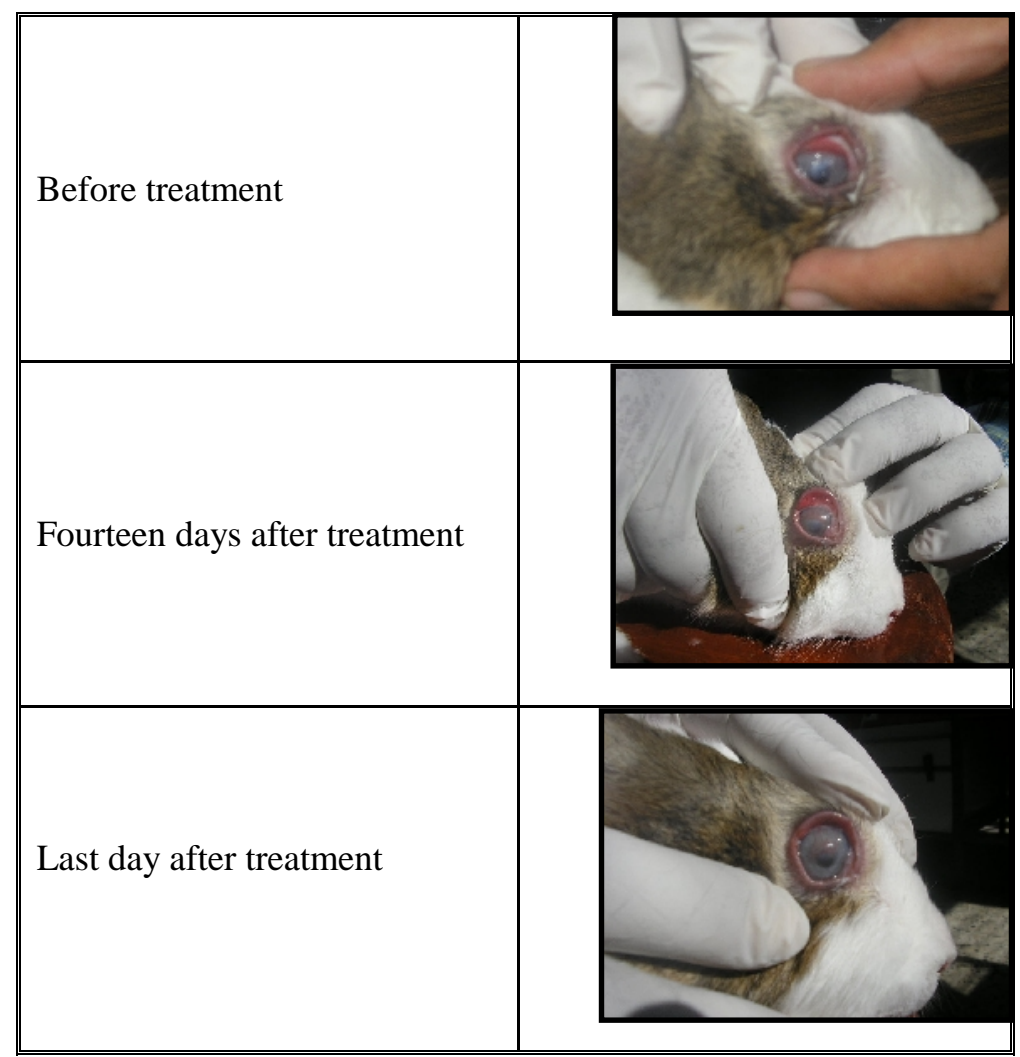

Fig. 4: Photographs showing gradual stages of healing and disappearance of Candida keratitis treated with fluconazole loaded neutral liposome composed of PC:Ch (7:4) (third group). 


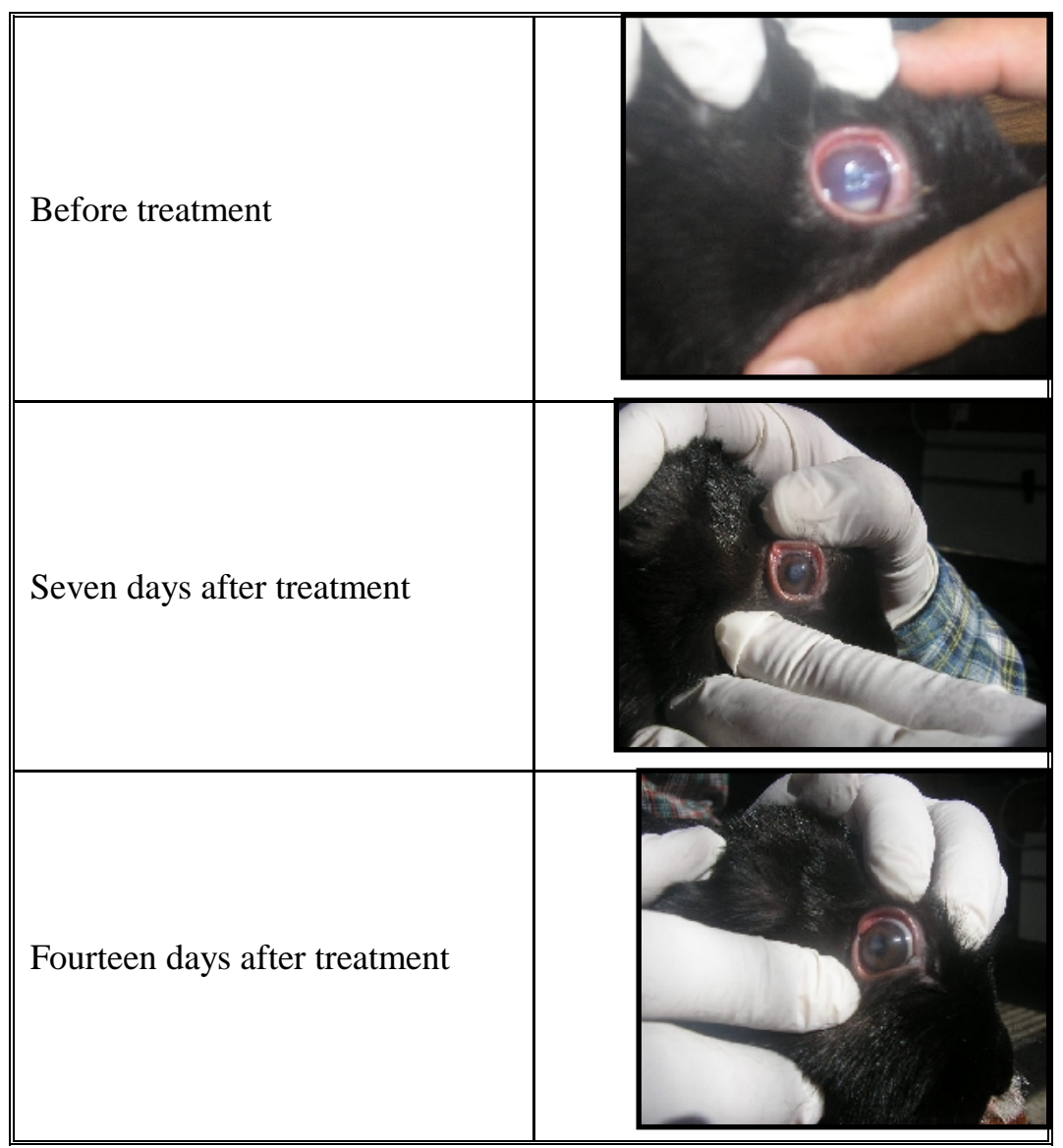

Fig. 5: Photographs showing gradual stages of healing and disappearance of Candida keratitis treated with fluconazole loaded negative liposome composed of PC:Ch:DP (5:5:1) (fourth group). 


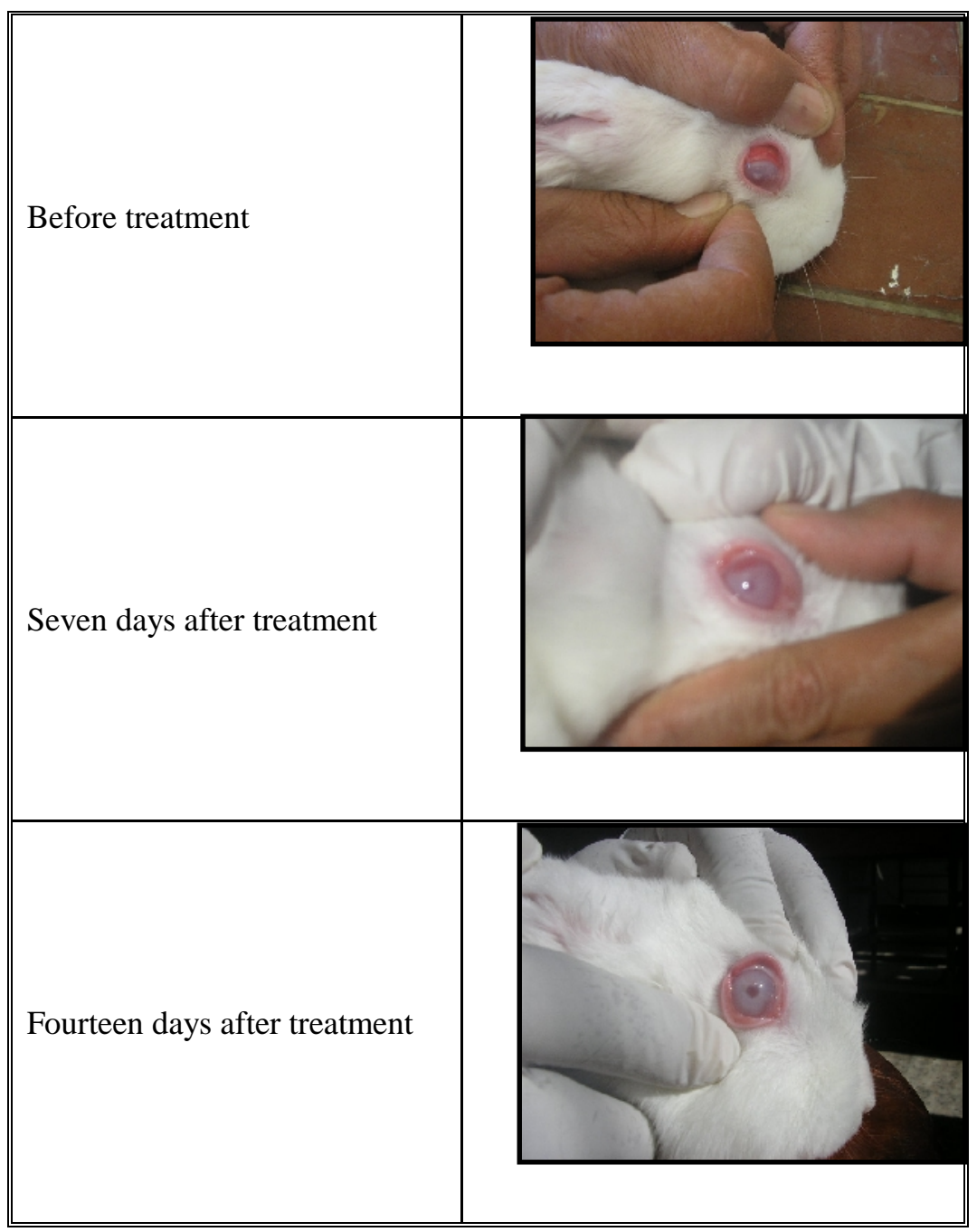

Fig. 6: Photographs showing gradual stages of healing and disappearance of Candida keratitis treated with fluconazole loaded positive liposome composed of PC:Ch:SA (5:5:0.5) (fifth group). 
Endophathalmitis occurred in control groups (receiving no medication) after 21 days (Fig. 7).

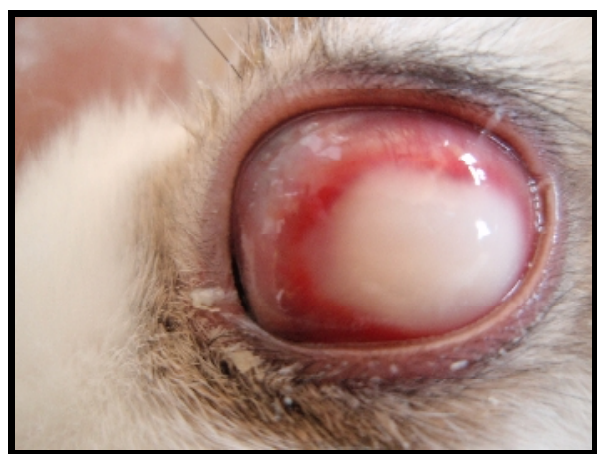

Fig. 7: Endophthalmitis control)

(positive

The drug was well tolerated by all animals. No adverse effects were observed. Statistical analysis of the differences between positive controls with regard to the number of residual ulcers showed a highly significant difference existing between the treated eyes and the controls $\left(\mathrm{p}=7.46 \times 10^{-7}\right)$. When comparing fluconazole liposomal formulations with fluconazole solution there was a statistically significant difference $(\mathrm{p}=$ 0.0052).

It was found that, fluconazole liposomal formulations were better than fluconazole solution this may be attributed to the viscosity of liposomal formulae which is higher than that of the solution. The higher viscosity may lead to an increase in the residence time than solution form $^{27}$. Also, it can be explained as, liposomal formulae produce sustained release effect into the eye or liposome can penetrate the corneal membrane and release fluconazole to exert its action.

Since, the cornea provided a negatively charged surface that should interact with the positively charged liposomes which may lead to an enhancement the effect of liposomes, however, the opposite was found and the negatively charged liposomes produced better effect than the positive one. There was no explanation found concerning this phenomenon, except that, the repulsion between the negatively charged liposomes and the negatively charged surface of the cornea resulted in disruption of the lipid bilayer and this in turn enhanced the release of the drug from liposomes giving a chance for the drug to be permeated through the corneal membranes.

The results also prove that the antifungal activity of fluconazole in liposomal formulation is better than fluconazole in solution. It was previously reported that, liposomal encapsulation increases the lipid solubility and hence their permeability through cell membrane. Moreover, the liposomes may enhance the binding of the drug to the active sites inside the cells ${ }^{28}$.

In the present study, one of the most important benefits of preparation of fluconazole in the form of liposomes is the decrease of the instillation frequency, onset and duration of recovery and healing from candidiasis. This gives a hope that, the incidence of other infections and complications that may occur due to the prolonged time required for the 
antifungal to start their effects may be diminished. The reduction of the recovery time may increase the patient compliance, and reduces the chance of the incidence of microbial resistance.

The above results showed that, the use of liposomes as a drug delivery system could contribute to the enhancement of the efficacy of fluconazole.

\section{REFERENCES}

1- I. P. Kaur, A. Garg, A. K. Singla and D. Aggarwal, Int. J. Pharm., 269, 1 (2004).

2- W. Behrens- Baumann, B. Klinge and R. Ruchel, Br. J. Ophthalmol., 74, 40 (1990).

3- A. Panda, N. Sharma and S. K. Angra, Cornea, 15, 373 (1996).

4- W. Schreiber, A. Olbrisch, C. K. Vorwerk, W. KÖnig and W. Behrens-Baumann, Invest. Ophthalmol. Vis. Sci., 44, 2634 (2003).

5- S. F. Urbak and T. Degn, Opthalmlogica, 208, 147 (1994).

6- D. S. Burgess, R. W. Hastings, K. K. Summers, T. C. Hardin and M. G. Rinaldi, Diagnostic Microbiology and Infectious Disease, 36, 13 (2000).

7- Goodman and Gilmans, The pharmacological basis of therapeutics, Macinillan Publishing Co., Inc., $9^{\text {th }}$ ed., 1996, pp 1175-1188.

8- P. Sandven, A. Bjomeklett and A. Maeland, Antimicrob. Agents Chemother., 37, 2443 (1993).
9- E. M. Johnson, D. W. Warnock, J. Luker, S. R. Porter and C. Scully, J. Antimicrob. Chemother., 35,103 (1995).

10- M. F. Price, M. T. LaRocco and L. O. Gentry, Antimicrob. Agents Chemother., 38, 422 (1994).

11- A. Ambrosini, G. D. Bossi, G. Dubinib, L. leone, M. Bossi and G. Zolese, Chemistry and Physics of Lipids, 95, 37 (1998).

12- J. Faergeman, J. Godleski, H. Laufen and R. H. Liss, Acta Derm. Veneorol. (Stockh), 75, 361 (1995).

13- R. W. Yee, S. M. Cheng, T. M. Ludden, J. E. Wallace and M. G. RinaldL, Cornea, 16, 64 (1997).

14- O. E. Abbasoglu, B. M. Hosal, B. Sener, N. Erdemoglu and E. Gursel, Exp. Eye Res., 72, 147 (2001).

15- O. N. El-Gazayerly and A. H. Hikal, Int. J. Pharm., 158, 121 (1997).

16- K. Singh and M. Mezei, ibid., 19, 263 (1984).

17- D. Meisner, J. Pringle and M. Mezei, ibid., 55, 105 (1989).

18- F. Suzoka and D. Papahadjopoulos, Proc. Natl. Acad. Sci., USA 75, 4194 (1978).

19- A. Bhatia, J. Pharm. Sci., 7, 252 (2004).

20- R. Agarwal and O. P. Katare, Pharmaceutical Technology, Nov. 2, 48 (2002).

21- W. Schreiber, A. Olbrisch, C. K. Vorwerk, W. KÖnig and W. 
Behrens-Baumann, Invest. Ophthalmol. Vis. Sci., 44, 2634 (2003).

22- W. Behrens-Baumann, Dev. Ophthalmol., 32, 27 (1999).

23- A. K. Al-Hussains, A. ElShanawany, E. A. Daef and M. M. Abd El-Latif, Bull. Ophthalmol Soc. Egypt., 90, 809 (1997).

24- W. Behrens- Baumann, B. Klinge and R. Ruchel, Br. J. Ophthalmol., 74, 40 (1990).

25- A. Panda, N. Sharma and S. K. Angra, Cornea, 15, 373 (1996).
26- D. Goldblum, B. E. Frueh, G. Sarra, K. Katsoulis and S. Zimmerli, Antimicrob. Agents and Chemotherapy, 49, 1359 (2005).

27- A. Bochot, E. Fattal, J. L. Grossiord, F. Puisieux and P. Couvreur, Int. J. Pharm., 162, 119 (1998).

28- Y. K. Oh, D. E. Nix and R. M. Straubinger, Antimicrob. Agents Chemother., 9, 2104 (1995). 